\title{
MODELACIÓN NO LINEAL DE MUROS DE MAMPOSTERÍA EMPLEANDO ELEMENTOS DE CONTACTO
}

\author{
José F. Lizárraga ${ }^{(1)}$ y Juan José Pérez Gavilán ${ }^{(2)}$
}

\begin{abstract}
RESUMEN
Se presenta la modelación de muros de mampostería simple y confinada sometidos a carga axial y lateral con el método de los elementos finitos para obtener la envolvente de resistencia a corte. Se utilizan elementos de contacto en las juntas para tomar en cuenta el comportamiento no lineal en la mampostería. Se tomaron en cuenta los dos tipos de falla más comunes: la falla en tensión (Modo I) y la falla a cortante (Modo II); en ambos casos se consideró el ablandamiento. En el rango elástico, se corroboró la consistencia de los resultados obtenidos con el modelo de elementos de contacto con los producidos con un modelo detallado y un modelo homogeneizado. En el rango no lineal, se utilizó un modelo trilineal de la superficie de fluencia que no incluye una reducción de la resistencia por el esfuerzo normal, a diferencia del esquema propuesto por otros autores que si la toman en cuenta. El efecto de esta consideración se estudió mediante la comparación de los resultados obtenidos con el modelo propuesto y los resultados numéricos y experimentales reportados en la literatura. Se comentan las principales diferencias. Finalmente, se modela un muro confinado ensayado en el laboratorio para comparar la envolvente de resistencia a corte experimental con la obtenida numéricamente. Los resultados obtenidos son bastante similares.
\end{abstract}

Palabras clave: mampostería; modelación; energía de fractura; resistencia lateral; curva envolvente

\section{NONLINEAR MODELLING OF MASONRY WALLS USING CONTACT ELEMENTS}

\begin{abstract}
Finite element modeling of unreinforced and confined masonry walls under axial and lateral loads is presented to estimate the shear strength envelope. Nonlinear contact elements are used at the joints to take into account the masonry's nonlinear behaviour. Two of the most common types of failure were considered at the joints: failure in tension (Mode I) and shear failure (Mode II); in each case softening were taken into account. Consistency of results obtained using contact elements and those obtained using detailed modeling and homogenized model was verified in the elastic range. In the non linear range, the yield surface was charcterized with a trilinear model that does not include a shear strength reduction due to normal stress, unlike other authors. The effect of this consideration was studied by comparing the results obtained with numerical and experimental results reported in

Artículo recibido el 20 de agosto de 2014 y aprobado para su publicación el 9 de diciembre de 2015. Se aceptarán comentarios y/o discusiones hasta cinco meses después de su publicación.

(1) Estudiante, Instituto de Ingeniería de la UNAM, Ciudad Universitaria, 04510, México; jlizarragap@iingen.unam.mx

(2) Investigador, Instituto de Ingeniería de la UNAM, Ciudad Universitaria, 04510, México; jipge@ @umas.iingen.unam.mx
\end{abstract}


the literature. The main differences are discussed. Finally, a confined masonry wall tested in the laboratory was modelled to compare the experimental strength envelope with the one obtained numerically. The results obtained are quite similar.

Keywords: masonry; modeling; fracture energy; lateral strength; envelope curve

\section{INTRODUCCIÓN}

El comportamiento sísmico de muros de mampostería puede ser estudiado realizando modelos de elementos finitos no lineales. Los modelos numéricos de elemento finito pueden ser calibrados a partir de resultados experimentales de pruebas de materiales y pruebas de muros con carga lateral y vertical. Un modelo numérico bien calibrado puede servir, en caso de ser necesario, para realizar estudios paramétricos que faciliten el desarrollo de modelos numéricos más simplificados que sirvan para el análisis y diseño sísmico de estructuras de mampostería.

Lourenço (1996) propuso una clasificación de los modelos de elementos finitos dependiendo del nivel de detalle de la modelación: Micro-modelo detallado en el que tanto el tabique como el mortero se representan con distintos elementos, mientras que la interfaz tabique-mortero es representada por elementos de interfaz. Estos modelos son ideales cuando se quiere un mejor comportamiento local pero se necesita de mucho esfuerzo computacional; Micro-modelo simplificado, en este modelo el tabique es representado por un solo material homogéneo, el comportamiento de la junta de mortero es representado con elementos de interfaz y Macro-modelo, en este modelo tanto el tabique y el mortero son sustituidos por un material homogéneo equivalente. La distribución de esfuerzos dentro del muro representa un valor promedio del esfuerzo en los distintos materiales.

Existen otro tipo de modelos numéricos, como por ejemplo el llamado análisis límite, en el cual se asumen ciertas hipótesis para conocer la carga máxima de una estructura. Orduña y Lourenço (2005) realizaron modelos considerando bloques rígidos para conocer la carga máxima que puede resistir un muro sin refuerzo cargado lateralmente con mampostería sin mortero. Casolo (2004) propone elementos rígidos unidos por resortes para modelar el comportamiento de la mampostería en el plano. Su principal objetivo con estos modelos fue el de tener un modelo con pocos grados de libertad y que describieran satisfactoriamente el comportamiento dinámico global en el rango elástico. Otro tipo de modelación es el método de los elementos distintos (Giamundo et al., 2014), en su trabajo evalúa la conveniencia en usar dicho método comparando con ejemplos con el método de los elementos finitos para representar el comportamiento de mampostería sin reforzar. Sarhosis et al. (2014) también utilizan este tipo de modelación para representar el comportamiento de muros diafragma de marcos de acero rellenos con mampostería. A continuación se mencionan algunos de los trabajos referentes a modelación con el método de los elementos finitos.

Mallick y Severn (1967) propusieron un método para calcular la rigidez de muros diafragma a carga lateral tomando en cuenta la separación entre el panel de mampostería y el marco de concreto. Para ello, utilizaron elementos de interfaz con cierto coeficiente de fricción y tensión igual a cero para modelar la distribución de esfuerzos entre el muro y el marco y la posible separación o deslizamiento en esa zona. Los resultados obtenidos fueron comparados con experimentos realizados en muros a escala con marcos de acero y utilizando una pasta especial para rellenar el marco. Una de las hipótesis hechas para modelar estructuras de más de un nivel fue que los muros tenían un comportamiento a corte despreciando los efectos de flexión.

King y Pandey (1978) realizaron un estudio similar al de Mallick y Severn, en el cual describen un procedimiento para determinar la rigidez de muros diafragma modelando la posible separación y 
deslizamiento utilizando elementos de interfaz con un determinado coeficiente de fricción. Una de sus mejoras respecto al modelo de Mallick y Severn fue que no consideraron los elementos del marco como axialmente rígidos, sino que se modelaron con elementos con tres grados de libertad por nodo. En este trabajo muestran los valores de la rigidez tangencial y coeficiente de fricción obtenidos para distintas interfaces entre el muro y el marco; además se hacen ver las limitaciones de modelar con diagonal equivalente este tipo de estructuras sometidas a cargas laterales y gravitacionales, pues las longitudes de contacto son notablemente mayores al tener cargas gravitacionales que si solo se tuvieran aplicadas cargas laterales.

Page (1978) fue el primero en utilizar elementos de interfaz para modelar el comportamiento no lineal de las juntas de mortero. Modeló los tabiques como elementos homogéneos elásticos isotrópicos, unidos por elementos de interfaz, en los cuales se incluye la falla en las juntas entre el mortero y los tabiques. Definió la superficie de falla en el espacio $(\sigma-\tau)$; esta superficie de falla la construyó con pruebas experimentales de pilas a compresión con juntas a distintos ángulos de inclinación. Dividió la superficie de falla en tres regiones. En la figura 1 se presenta la superficie utilizada por dicho autor. La región 1 representa la falla en tensión, la región 2 la falla por cortante puro, y la región 3 la falla combinada entre cortante y tensión en el tabique.

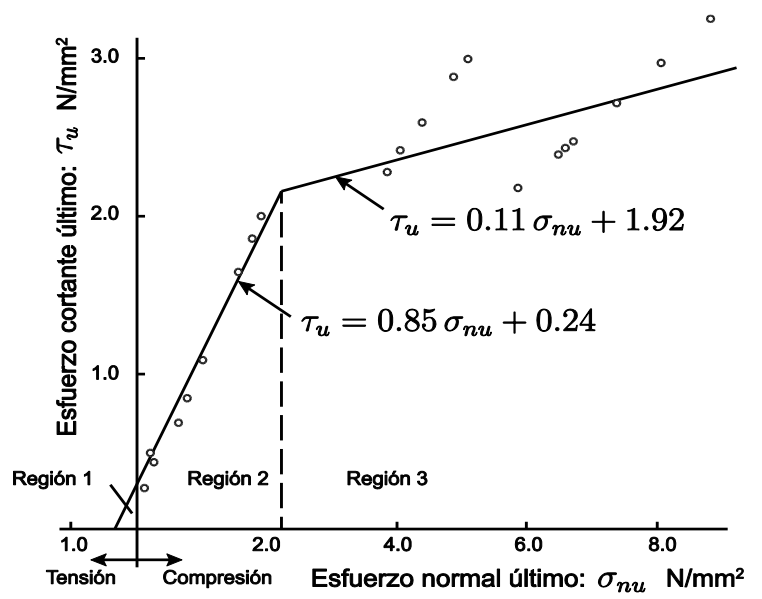

Figura 1. Superficie de falla en la interfaz de los tabiques (Page, 1978).

Dhanasekar y Page (1986) muestran que el comportamiento a carga lateral de un muro diafragma de mampostería no solo depende de la rigidez relativa entre el muro y el marco y su geometría, sino que también es influenciado por las propiedades a resistencia de la mampostería (en particular la magnitud de la resistencia a corte y tensión relativa a la resistencia a compresión). Ellos representaron a la mampostería con un material no lineal que tomó en cuenta la influencia de las juntas de mortero; utilizaron perfiles de acero como marco confinante, en el modelo, dicho marco se consideró elástico. Para definir el comportamiento de la mampostería a esfuerzos biaxiales, ensayaron paneles cuadrados de $360 \mathrm{~mm}$ realizando pruebas de compresión biaxial y tensión-compresión. De las pruebas de paneles establecieron las características de deformación y una superficie de falla en términos de los esfuerzos normales y paralelos a las juntas horizontales, y encontraron que el módulo de elasticidad resultó isotrópico en promedio; sin embargo, en el rango inelástico el comportamiento cambiaba sustancialmente con la orientación de los esfuerzos aplicados respecto a la inclinación de las juntas. Mediante el ensaye experimental de dos muros, uno cuadrado y uno rectangular observaron que el muro cuadrado falló paulatinamente por las juntas verticales y horizontales comenzando en el centro del muro y extendiéndose a las esquinas cargadas del muro. El muro rectangular falló a compresión por aplastamiento cerca de las esquinas cargadas. En su modelo lograron capturar el comportamiento de ambos muros con buena precisión hasta la carga máxima, y realizaron un estudio paramétrico con dos tipos de geometría utilizados en los experimentos y partiendo 
de las propiedades obtenidas de las pruebas de materiales. Se observó que para el muro cuadrado, la variación del módulo de elasticidad afecta solamente la rigidez del muro, mientras que para el muro rectangular, una variación del módulo de elasticidad de la mampostería cambia tanto la rigidez y la carga máxima. De éstos estudios paramétricos se hace ver que el aumentar la resistencia a tensión o a corte tiene un importante efecto en el nivel de carga máxima que alcanza el muro.

Liaw y Lo (1988) ensayaron y modelaron con elemento finito muros diafragma de concreto, sus modelos fueron a pequeña escala utilizando perfiles rectangulares de acero como marco confinante, probaron dos tipos de geometría, una con un panel y otra con dos paneles, para cada panel utilizaron tres dimensiones de columnas. Para modelar la interacción entre el marco de acero y el panel de concreto utilizaron elementos de interfaz. Para esfuerzos de tensión consideraron tanto la rigidez normal y tangencial igual a cero, mientras que para esfuerzos de compresión la rigidez normal se tomó como infinitamente rígida y la fuerza cortante se tomó en cuenta por fricción. El panel se modeló como un material frágil a tensión con una pendiente inicial, y a compresión consideraron una relación esfuerzo-deformación no lineal. El material se considera isotrópico antes del agrietamiento, mientras que después del agrietamiento el módulo de elasticidad y el módulo de cortante a lo largo de la grieta se tomaron igual a cero; el módulo de elasticidad perpendicular a la grieta no cambia. El acero utilizado en el marco se consideró como elastoplástico. En su trabajo no reportan las propiedades de los materiales utilizados. Todos sus modelos fallaron después de la formación de articulaciones plásticas precedidas por aplastamiento en las esquinas cargadas del muro, conforme aumentaban el tamaño de las columnas se observa en sus estudios que disminuía la concentración de esfuerzos cercana a las esquinas cargadas.

König (1991) muestra investigaciones desarrolladas en la universidad de Darmstadt de temas relacionados con ingeniería sísmica. En uno de sus ejemplos describe el trabajo por Schmidt (1991) en el cual modela con el método de los elementos finitos tres marcos de concreto reforzado, de los cuales dos son muros diafragma de mampostería. Para ello, utilizó elementos membrana para modelar el panel de mampostería y el marco de concreto, el refuerzo a flexión fue representado con elementos barra y se implementó refuerzo distribuido a corte para las columnas y las vigas. Se utilizaron elementos interfaz para representar la interacción entre el marco y el muro. König estudió dos tipos de muros, uno tenía una rigidez pequeña en la interfaz entre el muro y el marco, y el otro tenía junta de mortero en dicha interfaz. La mampostería la consideró con un solo material con diferentes propiedades a compresión en la dirección paralela y normal a las juntas horizontales. Se hace ver que la rigidez lateral del muro puede modelarse con bastante aproximación hasta el $60-80 \%$ de la carga última, luego incrementa la discrepancia respecto al ensaye experimental, esta diferencia la atribuyen al hecho de que los ciclos de carga influyen en el comportamiento del material, el cual no puede ser representado correctamente con dicho modelo. Se concluye que el agrietamiento obtenido en el modelo y el observado experimentalmente es similar.

Asteris y Tzamtzis (2003) presentan la modelación de muros diafragma de mampostería de un panel y con varios niveles. Su principal objetivo fue encontrar las longitudes de contacto entre el muro y el marco como una solución integral del modelo, y conocer la distribución de las fuerzas cortantes en las columnas que ocasionan el tener los muros dentro de los marcos de concreto para distintas geometrías.

Autores como Lofti y Shing (1994) han utilizado la modelación con elementos de interfaz para modelar muros diafragma de mampostería con marcos de concreto reforzado, en su modelo representan el comportamiento a tensión y cortante en la unión entre tabique y mortero con una superficie de falla hiperbólica; incluyen la falla a tensión y a compresión en el tabique utilizando un modelo de agrietamiento distribuido. Stavridis y Shing (2010) presentaron una versión más reciente del modelo citado anteriormente, en su trabajo consideran la falla por corte en las columnas con elementos de interfaz; además, dan recomendaciones para la calibración de las propiedades de los materiales y también realizan un estudio paramétrico para conocer la sensibilidad de los parámetros en el comportamiento global del muro. De los 
análisis observaron que las propiedades del mortero tienen una mayor influencia en el comportamiento del muro.

Lourenço (1996) utiliza un modelo de interfaz compuesto con propiedades no lineales donde se incluye la falla a tensión, falla a cortante y falla a compresión. Las piezas de mampostería permanecen elásticas, solo se incluye la falla a tensión en la mitad de la pieza. El autor considera que la falla a tensión diagonal está incluida en la zona de falla a compresión limitando el esfuerzo cortante resistente en la interfaz. Oliveira (2003) realiza una extensión del modelo de Lourenço (1996), extendió el modelo de interfaz compuesto para poder modelar el comportamiento de estructuras de mampostería en cargas cíclicas. Van Zijl (2004) propone un modelo para representar el ángulo de dilatación en las interfaces, el cual complementa el modelo propuesto por Lourenço (1996).

Para conocer la resistencia de un muro sometido a carga cíclica reversible se tienen modelos en los que se predice también la energía histerética disipada (Gambarotta y Lagomarsino, 1997). Se considera el ablandamiento del material e introducen para tal efecto la energía de fractura a tensión y cortante.

En México también se han realizado esfuerzos para la modelación de muros de mampostería utilizando elementos finitos. Bazán (1980) propone inicialmente un modelo de material con propiedades elásticas isotrópicas, en un análisis incremental, revisa elemento por elemento los distintos límites de resistencia, y en caso de detectar una falla calcular las fuerzas que deben redistribuirse en el resto de la estructura (fuerzas de transferencia). En su programa puede variar la resistencia a tensión diagonal a diferentes ángulos, estos valores de resistencia a tensión los obtuvo de resultados experimentales. Orduña (1999) modeló la mampostería con un criterio de fluencia combinado de Von Mises para compresión y de Rankine para tensión; además, se incluyó el ablandamiento a compresión y a tensión haciendo una calibración de dichos parámetros. Lizárraga (2011) y Lizárraga y Pérez-Gavilán (2011) utilizaron modelos de plasticidad con elementos finitos para simular el comportamiento de muros ante cargas laterales hasta la falla, utilizaron un modelo de material homogéneo equivalente por medio del criterio de falla DruckerPrager.

Para poder definir los parámetros que utilizan los modelos no lineales de elementos finitos es necesario realizar pruebas especializadas de laboratorio. Las propiedades mecánicas de la mampostería que se han obtenido en México (Meli y Reyes, 1975) son principalmente referentes a la resistencia máxima a compresión de pilas, muretes a compresión diagonal y pruebas a cortante; sin embargo, faltan resultados experimentales que describan el comportamiento de la mampostería después de la carga máxima tanto en resistencia como en deformación. Lizárraga y Pérez Gavilán (2013) realizaron pruebas en mampostería de block de concreto multiperforado, en esa investigación obtuvieron los principales parámetros para poder calibrar modelos como los propuestos por Lourenço (1996) y Van Zijl (2004).

\section{Comportamiento de la mampostería}

El comportamiento de la mampostería sometida a tensión, compresión o cortante, se suele caracterizar con la curva carga-desplazamiento. Los parámetros más utilizados para describir dicha curva en cada caso son: la pendiente del segmento aproximadamente recto que va desde carga nula hasta la carga máxima, la carga resistente máxima y el desplazamiento para el cual se alcanza la resistencia máxima. El comportamiento después de la resistencia máxima conocido como el ablandamiento del material, puede describirse con la energía de fractura, la cual está definida como la integral de la curva esfuerzodesplazamiento plástico. El proceso de ablandamiento para la falla a tensión (Modo I) puede representarse con la energía de fractura en tensión $G_{f}^{I}$. El comportamiento constitutivo de la mampostería a tensión tiene, por lo general, una forma exponencial (ver figura 2a); sin embargo, puede utilizarse una aproximación lineal (ver figura 3 ) sin cometer un error significativo. 


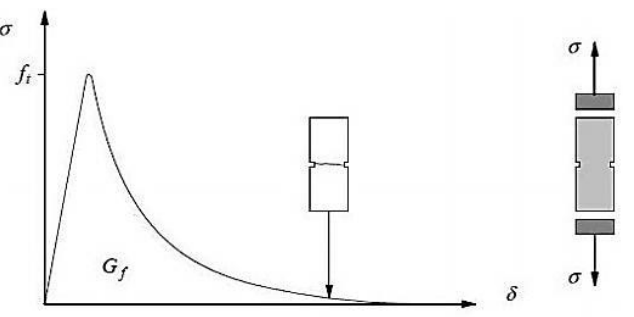

a) Prueba a tensión uniaxial

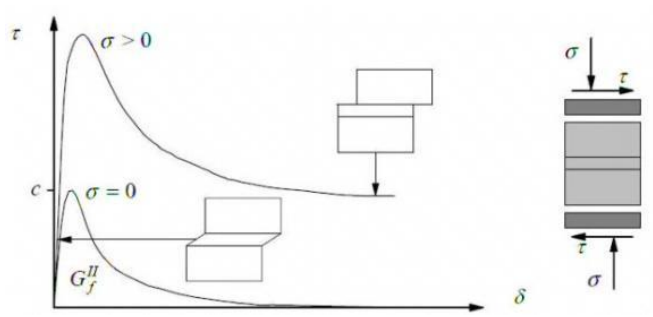

b) Prueba a corte con distintos niveles de carga axial

Figura 2. Comportamiento típico de materiales cuasi-frágiles y definición de energía de fractura (Lourenço, 1996).

Si se utiliza el modelo propuesto por Lourenço (1996) para representar la falla por deslizamiento en las juntas, el proceso de ablandamiento consiste en una degradación de la cohesión y el coeficiente de fricción del modelo. La falla por deslizamiento (Modo II) ocurre cuando se supera la resistencia a cortante $\tau_{1}$ (ecuación 1.a) representada por la ley de Mohr-Coulomb siempre que el esfuerzo normal sea menor a un cierto valor $\sigma_{i}$ (positivo a compresión), a partir del cual la resistencia a corte $\tau_{2}$ empieza a reducirse de forma elíptica al aumentar el esfuerzo de compresión (ecuación 1.b). En la ecuación $1 c$ es la cohesión, $\mu$ es el coeficiente de fricción de la superficie de contacto (tangente del ángulo de fricción $\phi$ ), $\sigma_{n}$ el esfuerzo de confinamiento, $f_{m}$ es la resistencia a compresión de la mampostería y $C_{S}$ controla la contribución del esfuerzo cortante a la falla a compresión. El comportamiento inelástico a cortante puede ser descrito por la energía de fractura en modo II $\left(G_{f}^{I I}\right)$, la cual se calcula, como en el caso de tensión, con la integral de la curva esfuerzo cortante-desplazamiento plástico.

Mediante pruebas a cortante con distintos niveles de confinamiento se pueden construir curvas como las que aparecen en la figura $2 \mathrm{~b}$. Se observa que al aumentar la carga de confinamiento se eleva también la energía de fractura a corte $G_{f}^{I I}$; se puede obtener la energía de fractura en función del nivel de confinamiento a partir de una relación lineal (Pluijm, 1993; Augenti y Parisi, 2011) con la forma que aparece en la ecuación 2. En la ecuación 2, $a$ y $b$ son constantes que se obtienen al ajustar, a partir de una relación lineal, la dependencia de la energía de fractura con el nivel de confinamiento $\left(\sigma_{n}\right)$.

$$
\left\{\begin{array}{cc}
\tau_{1}=\mu \sigma_{n}+c & \sigma_{n}<\sigma_{i} \\
\tau_{2}=\sqrt{\frac{1}{C_{s}}\left(f_{m}^{2}-\sigma_{n}^{2}\right)} & \sigma_{n} \geq \sigma_{i}
\end{array}\right.
$$

donde $\sigma_{i}$ es tal que $\tau_{1}\left(\sigma_{n}=\sigma_{i}\right)=\tau_{2}\left(\sigma_{n}=\sigma_{i}\right)$

$$
G_{f}^{I I}=a+b \sigma_{n}
$$

\section{MODELO PROPUESTO}

Al modelo propuesto se le llamará, para efectos de comparación con otros modelos, como micromodelo simplificado propuesto. La modelación se realizó en el programa de elementos finitos Ansys v13.0. El modelo consiste en asignar elementos de contacto en las juntas. Las propiedades de la mampostería se consideran elásticas, y el comportamiento no lineal se asigna a los elementos de contacto. Se permite la falla 
a tensión y a cortante en juntas horizontales y verticales. El micro-modelo simplificado propuesto es una adaptación del modelo presentado por Lourenço (1996). Se considera a las piezas como elementos elásticos unidos por elementos interfaz con cierta rigidez normal y tangencial que tratan de representar la rigidez normal y tangencial de la junta.

Para determinar las rigideces normal $k_{n}$ y tangencial $k_{s}$ se utilizan las ecuaciones 3 y 4 (Lourenço, 1996). En estas ecuaciones $h_{m}$ es el espesor de la junta de mortero, $E_{u}$ y $E_{m}$ son el módulo de elasticidad de la pieza y del mortero, mientras que $G_{u}$ y $G_{m}$ son el módulo de cortante de la pieza y del mortero respectivamente. El módulo de elasticidad que se introduce es el que corresponde a la dirección perpendicular a la junta que se está modelando.

$$
\begin{aligned}
& k_{n}=\frac{E_{u} E_{m}}{h_{m}\left(E_{u}-E_{m}\right)} \\
& k_{s}=\frac{G_{u} G_{m}}{h_{m}\left(G_{u}-G_{m}\right)}
\end{aligned}
$$

\section{Falla en modo I}

Se utilizó un modelo de material bilineal para representar el ablandamiento en tensión. En el programa utilizado, este tipo de modelo es llamado modelo de zona cohesiva CZM (Ansys v13) y está basado en el modelo propuesto por (Alfano y Crisfield, 2001). Este tipo de material define el modo de separación normal entre los contactos. En la figura 3 se observa que inicialmente se tiene una pendiente elástica, hasta un esfuerzo de tensión máxima $f_{t}$, para luego presentar un ablandamiento lineal el cual se puede representar con la energía de fractura a tensión $G_{f}^{I}$ como se muestra en la figura 3. Debido a que el ablandamiento depende sólo de las deformaciones plásticas $\delta_{p}$, la energía de fractura se puede representar con la ecuación 5 , en la figura 3 se muestran los parámetros utilizados.

$$
G_{f}^{I}=\frac{1}{2} f_{t} \delta_{p}
$$

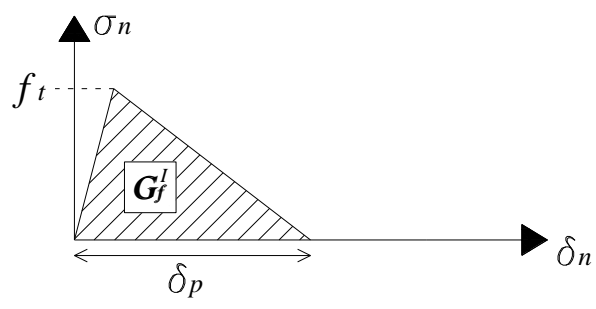

Figura 3. Gráfica esfuerzo normal-deformación normal para material con comportamiento bilineal.

\section{Falla en modo II}

Se utilizó el criterio de falla de Mohr-Colomb (ecuación 1), considerando el ablandamiento con un modelo trilineal (ver figura 4a) mediante el modelo de material llamado FRIC (Ansys v13). En la figura 4a, $c$ es la cohesión, $\tau_{m}$ y $\tau_{r}$ son la resistencia a corte máxima y residual dado un nivel de confinamiento $\sigma_{n}, \mathrm{y}$ por último $s$ es la deformación tangencial plástica que existe al pasar linealmente de $\tau_{m}$ a $\tau_{r}$. La energía de fractura a corte $G_{f}^{I I}$ estará definida como el área bajo la curva esfuerzo-desplazamiento plástico que existe al pasar de $\tau_{m}$ a $\tau_{r}$. Este modelo no considera la degradación de la cohesión, por lo que ésta se mantiene constante y lo único que se degrada es el coeficiente de fricción, pasando del coeficiente de fricción inicial $\mu$ al coeficiente de fricción residual $\mu_{r}$, por tanto $\tau_{m} \mathrm{y} \tau_{r}$ se definen con las ecuaciones 6 y 7 . 


$$
\begin{gathered}
\tau_{m}=c+\mu \sigma_{n} \\
\tau_{r}=c+\mu_{r} \sigma_{n}
\end{gathered}
$$

Debido a que no existe degradación de la cohesión es necesario definir el valor de $\mu_{r}$ tal que $\tau_{r}$ (ecuación 7) de un valor igual a $\tau_{r 0}$, donde $\tau_{r 0}$ es el cortante residual con $c=0$ (ecuación 8); $\mu_{r 0}$ es el valor del coeficiente de fricción residual con $c=0$. Para obtener lo expuesto anteriormente, se igualan las ecs. 7 y 8 y se obtiene la ecuación 9. Si el producto $\mu_{r 0} \sigma_{n}>c$, el mínimo valor para $\mu_{r}$ es $\mu_{r}=0$.

$$
\begin{aligned}
& \tau_{r 0}=\mu_{r 0} \sigma_{n} \\
& \mu_{r}=\frac{\mu_{r 0} \sigma_{n}-c}{\sigma_{n}}
\end{aligned}
$$

El parámetro $s$ se determina considerando que el área bajo la curva esfuerzo-deformación plástica que se obtiene al pasar de $\tau_{m}$ a $\tau_{r}$ en una distancia $s$ para un nivel de confinamiento $\sigma_{n}$, es igual a la energía de fractura a corte para ese nivel de confinamiento. Por tanto la energía de fractura se obtiene con la ecuación 10. El parámetro $s$ se obtiene con la ecuación 11, que resulta de sustituir las ecuaciones 6 y 7 en la ecuación 10.

$$
\begin{aligned}
& G_{f}^{I I}=\frac{s\left(\tau_{m}-\tau_{r}\right)}{2} \\
& s=\frac{2 G_{f}^{I I}}{\sigma_{n}\left(\mu-\mu_{r}\right)}
\end{aligned}
$$

Debido a que se necesita establecer un valor máximo de resistencia a corte, éste se toma en cuenta con el parámetro $\tau_{u}$, quedando la superficie de falla como se muestra en la figura $4 \mathrm{~b}$. Como una aproximación se tomará el valor de $\tau_{u}$ (ecuación 12) igual al valor máximo de cortante que se desarrolla en una superficie de falla elíptica (ecuación 1.b).

$$
\tau_{u}=\frac{f_{m}}{\sqrt{C_{s}}}
$$

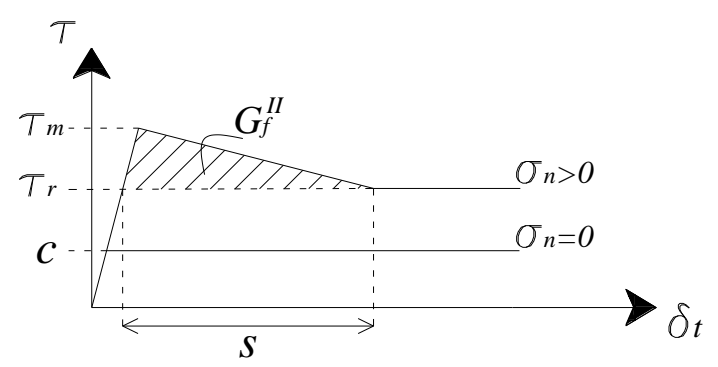

a) Modelo trilineal para la falla en modo II

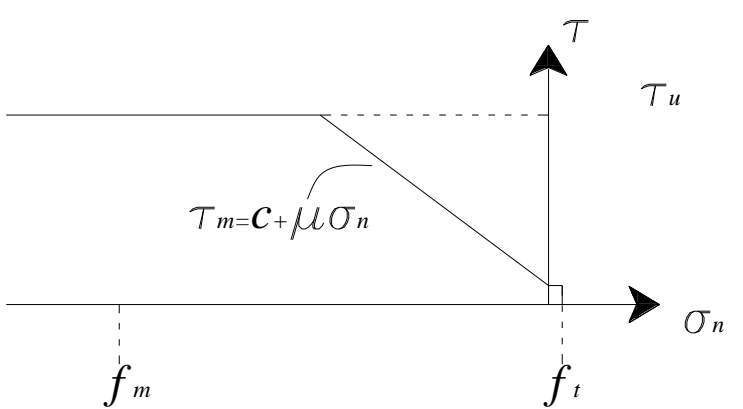

b) Superficie de falla inicial utilizada

Figura 4. Falla a cortante y superficie de falla utilizada en el modelo propuesto. 


\section{MODELOS DE ELEMENTO FINITO REALIZADOS}

\section{Modelo elástico de muros de mampostería simple}

Para conocer el comportamiento elástico de los diferentes niveles de modelación, se estudió el muro de la figura 5 que corresponde a los muros J4D/J5D y J7D presentados por Lourenço (1996). Las propiedades elásticas de los materiales se muestran en la tabla 1. Las dimensiones de los tabiques son 210 $\mathrm{mm}$ de largo, $52 \mathrm{~mm}$ de altura y $100 \mathrm{~mm}$ de espesor; el espesor de la junta de mortero es de $10 \mathrm{~mm}$. Se compararon los resultados obtenidos con los distintos niveles de modelación: micro-modelo detallado, micro-modelo simplificado y modelo homogéneo. En el micro-modelo detallado se modelaron tanto las piezas como el mortero con distintos elementos. En el micro-modelo simplificado se utilizaron elementos continuos para los tabiques y elementos de interfaz para las juntas. Para el micro-modelo detallado y simplificado se utilizaron las propiedades reportadas en la referencia antes mencionada. Para el modelo homogéneo, las propiedades elásticas se obtuvieron utilizando el método descrito en Lizárraga (2011). Las propiedades elásticas aparecen en la tabla 1.

Tabla 1. Propiedades elásticas para los diferentes tipos de modelos de los muros J4D/J5D y J7D

\begin{tabular}{|c|c|c|c|}
\hline \multicolumn{2}{|c|}{ Micro-modelo detallado } & \multicolumn{2}{|c|}{ Micro-modelo simplificado } \\
\hline Pieza & $\begin{array}{c}E_{u}=16,700 \mathrm{~N} / \mathrm{mm}^{2} \\
v=0.15\end{array}$ & Pieza & $\begin{array}{c}E_{u}=16,700 \mathrm{~N} / \mathrm{mm}^{2} \\
v=0.15\end{array}$ \\
\hline Mortero & $\begin{array}{c}E_{m}=782 \mathrm{~N} / \mathrm{mm}^{2} \\
v=0.15\end{array}$ & $\begin{array}{l}\text { Mitad de tabiques } \\
k_{n}=10^{6} \mathrm{~N} / \mathrm{mm}^{3} \\
k_{s}=10^{6} \mathrm{~N} / \mathrm{mm}^{3}\end{array}$ & $\begin{array}{l}\text { Unión de tabiques } \\
k_{n}=82 \mathrm{~N} / \mathrm{mm}^{3} \\
k_{S}=36 \mathrm{~N} / \mathrm{mm}^{3}\end{array}$ \\
\hline \multicolumn{4}{|c|}{ Modelo homogéneo } \\
\hline$E_{x}$ & $E_{y}$ & $G_{x y}$ & $v_{x y}$ \\
\hline $8,515 \mathrm{~N} / \mathrm{mm}^{2}$ & $3,924 \mathrm{~N} / \mathrm{mm}^{2}$ & $1,533 \mathrm{~N} / \mathrm{mm}^{2}$ & 0.012 \\
\hline
\end{tabular}

El muro de la figura 5 fue sujeto a un desplazamiento lateral de $0.5 \mathrm{~mm}$ en su parte superior con el giro restringido. En la figura 6 se muestran los resultados del esfuerzo cortante utilizando los niveles de modelación mencionados. Se compara la distribución de cortante a lo largo del muro (ver figura 6). Para el modelo homogéneo se tomó una sección a la mitad de la altura del muro para obtener el esfuerzo cortante, para los micro-modelos se tomó una sección entre la octava y la novena hiladas al centro del tabique para obtener sus correspondientes esfuerzos cortantes.

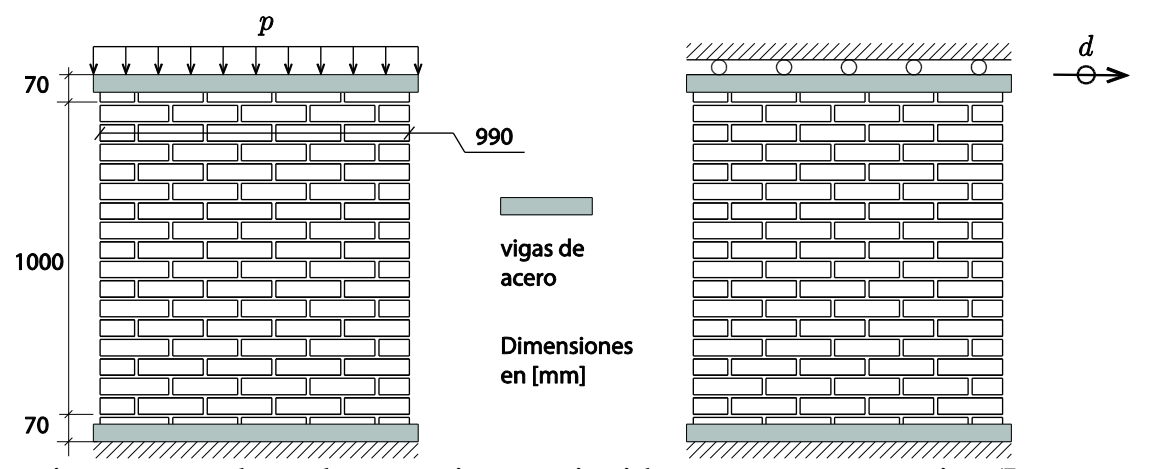

Figura 5. Muro sujeto a carga lateral y con giro restringido en su parte superior (Lourenço, 1996). 


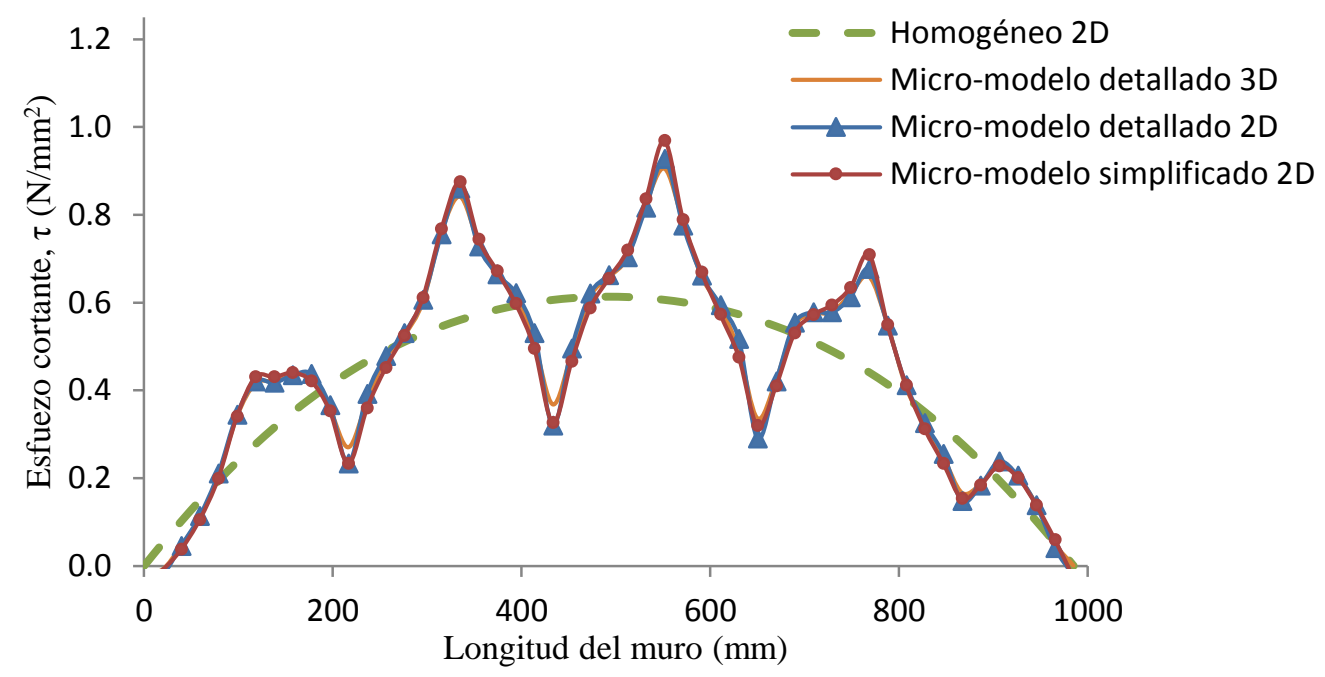

Figura 6. Comparación del esfuerzo cortante a lo largo del muro.

Se compararon además los resultados obtenidos con elementos sólidos (3D) y con elementos de esfuerzo plano (2D) (ver figura 6). Se observó que no existen diferencias significativas utilizando elementos sólidos de 8 nodos (SOLID185) con dimensiones medias de $10 \mathrm{~mm}$ y con elementos de esfuerzo plano de 8 nodos (PLANE183) también de dimensiones de $10 \mathrm{~mm}$, por tanto para el resto de los casos estudiados se utilizaron elementos de esfuerzo plano de dimensión aproximada de $10 \mathrm{~mm}$ a excepción del micro-modelo simplificado (2D), en el que se utilizó una malla de $30 \mathrm{~mm}$.

En la figura 7 se muestra gráficamente los resultados obtenidos para los diferentes niveles de modelación, se muestran los esfuerzos cortantes $(\tau)$ y el esfuerzo principal a compresión $\left(\sigma_{\min }\right)$. En la figura 7a.1 se observa que existen concentraciones de esfuerzo cortante a la mitad del muro y en medio de las piezas, esto se pudo representar también con el micro-modelo simplificado (ver figura 7b.1). La figura 7a.2 muestra que se presentan diagonales de compresión que pasan aproximadamente por la mitad de los tabiques, esto también se observó en el micro-modelo simplificado (ver figura 7b.2). En el modelo homogéneo no se observan las concentraciones de esfuerzo que se describieron, sino que el cortante tiene un valor máximo al centro del muro (ver figura 7c.1), y se presenta sólo una diagonal de compresión (ver figura $7 \mathrm{c} .2)$.

\section{Modelo no lineal de muros de mampostería simple}

Se modelaron dos de los muros presentados en Lourenço (1996) con las dimensiones que se muestran en la figura 5. Los muros fueron sometidos a desplazamiento lateral incremental en su parte superior restringiendo el giro. Se utilizó el micro-modelo simplificado propuesto (con elementos de contacto). Se modeló un muro con carga axial de $0.3 \mathrm{~N} / \mathrm{mm}^{2}$, se cuentan con dos resultados experimentales para este nivel de carga axial (J4D y J5D); el segundo muro se modeló con carga axial de $2.2 \mathrm{~N} / \mathrm{mm}^{2}$ (MJ7D). Los resultados experimentales de dichos muros fueron reportados por Raijmakers y Vermeltfoort (1992) y Vermeltfoort y Raijmakers (1993).

Los elementos utilizados para representar a las piezas fueron elementos de esfuerzo plano (PLANE183) de 8 nodos con dos grados de libertad por nodo (translación en los ejes x,y). Para modelar los contactos se utilizaron elementos 2D (CONTA172 y TARGE169) de tres nodos con dos grados de libertad por nodo (translación en los ejes x,y). 

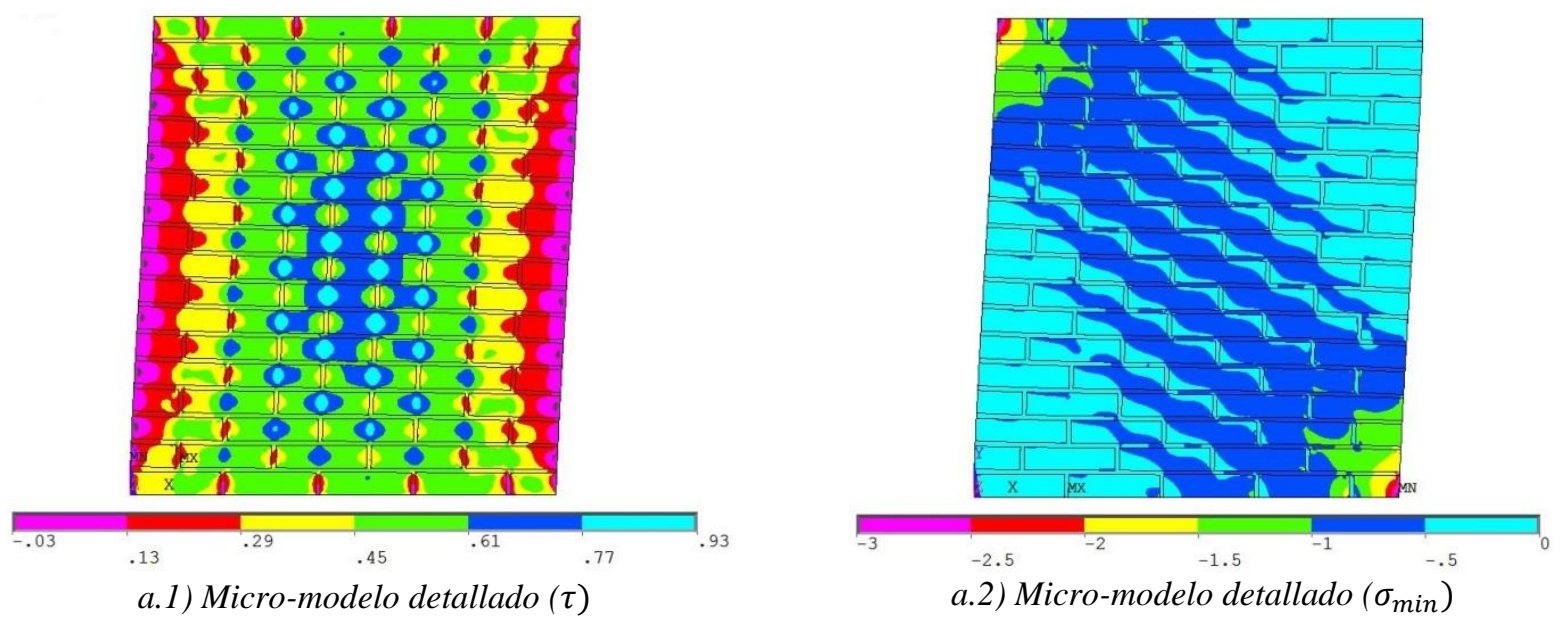

a.2) Micro-modelo detallado $\left(\sigma_{\min }\right)$

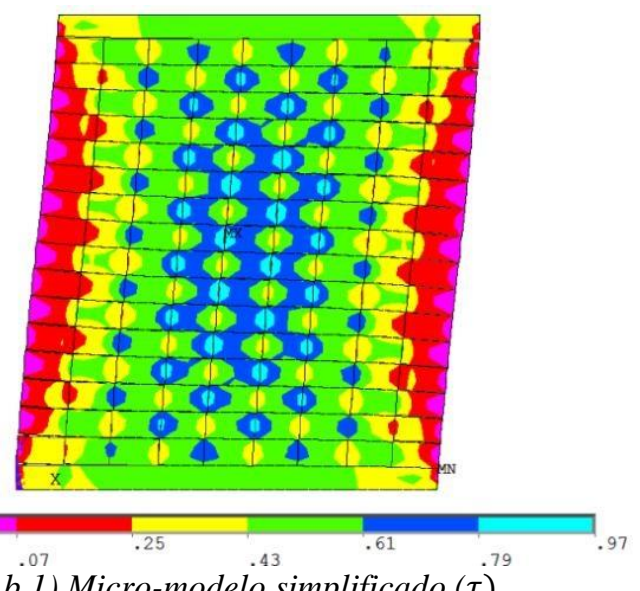

b.1) Micro-modelo simplificado $(\tau)$

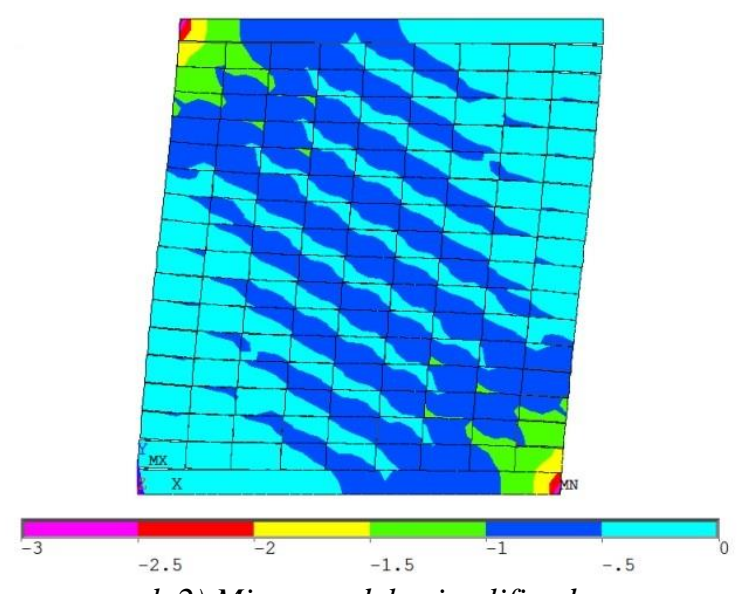

b.2) Micro-modelo simplificado
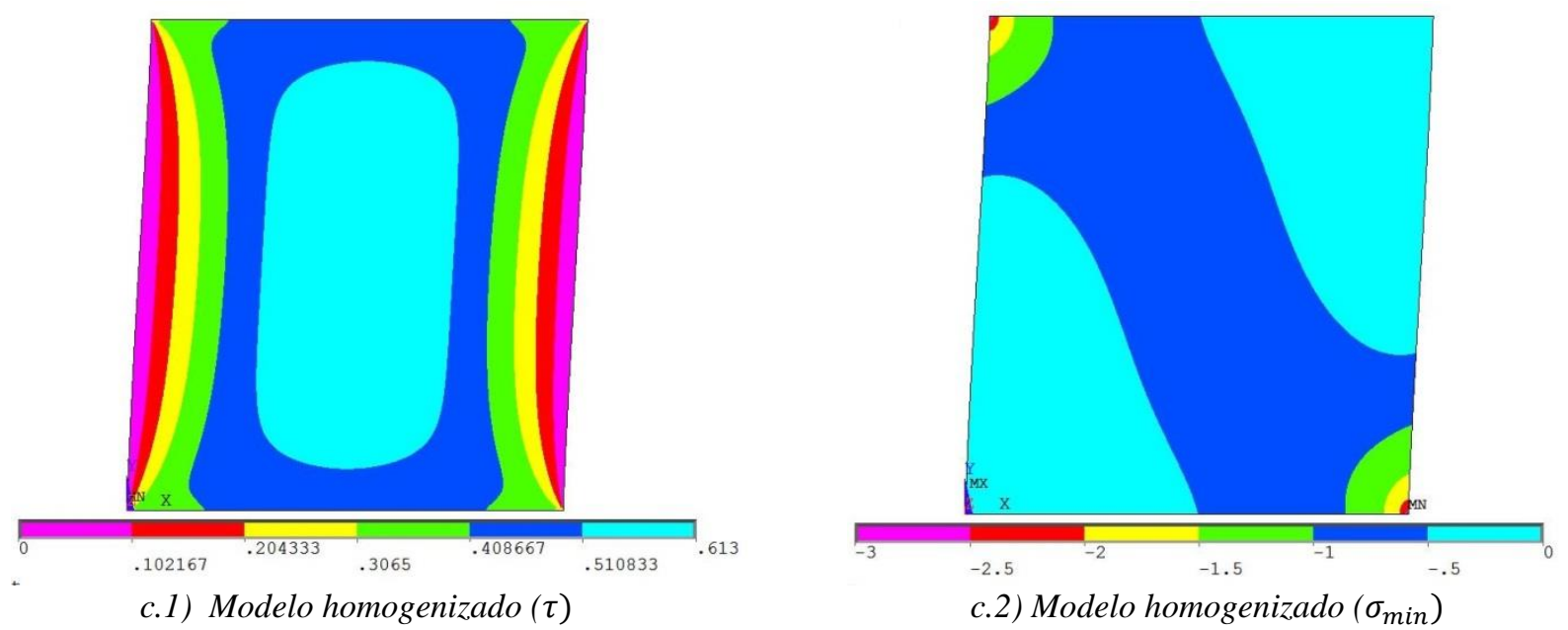

Figura 7. Representación gráfica de esfuerzos cortantes $\tau\left(\mathrm{N} / \mathrm{mm}^{2}\right)$ y esfuerzo principal a compresión $\sigma_{\min }\left(\mathrm{N} / \mathrm{mm}^{2}\right)$ : a) Micro-modelo detallado; b) Micro-modelo simplificado; c) Modelo homogéneo. 
Las propiedades elásticas se muestran en la tabla 1, las propiedades inelásticas asignadas se resumen en la tabla 2. Estas propiedades se obtuvieron de lo reportado por Lourenço (1996). El coeficiente de fricción residual para ambos muros fue de $\mu_{r 0}=0.75 ; G_{f}^{I I}=0.125 \mathrm{Nmm}^{-1}$ para los muros J4D/J5D; $G_{f}^{I I}=$ $0.05 \mathrm{Nmm}^{-1}$ para el muro J7D; $f_{m}=10.5 \mathrm{~N} / \mathrm{mm}^{2}$ para los muros $\mathrm{J} 4 \mathrm{D} / \mathrm{J} 5 \mathrm{D} ; f_{m}=11.5 \mathrm{~N} / \mathrm{mm}^{2}$ para el muro J7D y $C_{s}=9$ para ambos muros.

Tabla 2. Propiedades inelásticas para los muros J4D/J5D y J7D

\begin{tabular}{|c|c|c|c|c|c|c|c|c|c|}
\hline \multirow[t]{2}{*}{ Muro } & \multicolumn{2}{|c|}{$\begin{array}{c}\text { Tensión } \\
\text { (Mitad de tabiques) }\end{array}$} & \multicolumn{2}{|c|}{$\begin{array}{l}\text { Tensión } \\
\text { (Juntas) }\end{array}$} & \multicolumn{5}{|c|}{$\begin{array}{l}\text { Cortante } \\
\text { (Juntas) }\end{array}$} \\
\hline & $\begin{array}{c}f_{t} \\
\mathrm{~N} / \mathrm{mm}^{2}\end{array}$ & $\begin{array}{c}G_{f}^{I} \\
\mathrm{Nmm}^{-1}\end{array}$ & $\begin{array}{c}f_{t} \\
\mathrm{~N} / \mathrm{mm}^{2} \\
\end{array}$ & $\begin{array}{c}G_{f}^{I} \\
\mathrm{Nmm}^{-1} \\
\end{array}$ & $\begin{array}{c}c \\
\mathrm{~N} / \mathrm{mm}^{2} \\
\end{array}$ & $\begin{array}{l}\mu \\
-\end{array}$ & $\begin{array}{c}\mu_{r}(e c .9) \\
-\end{array}$ & $\begin{array}{c}s(e c .11) \\
\mathrm{mm}\end{array}$ & $\begin{array}{c}\tau_{u}(e c .12) \\
\mathrm{N} / \mathrm{mm}^{2}\end{array}$ \\
\hline $\mathrm{J} 4 \mathrm{D} / \mathrm{J} 5 \mathrm{D}$ & 2 & 0.08 & 0.25 & 0.018 & 0.35 & 0.75 & 0 & 1.11 & 3.5 \\
\hline J7D & 2 & 0.08 & 0.16 & 0.012 & 0.224 & 0.75 & 0.648 & 0.45 & 3.83 \\
\hline
\end{tabular}

En la figura 8 se presentan los resultados obtenidos en este estudio, los obtenidos experimentalmente (Exp) y los obtenidos numéricamente por Lourenço (1996). En las figuras 9 y 10 se muestran los estados de esfuerzos principales obtenidos en este estudio y lo reportado por dicho autor. Como un análisis adicional se modeló la falla por tensión a la mitad del tabique; sin embargo, no se encontraron diferencias significativas entre las curvas cálculadas sin incluir esta falla, por lo que en el micro-modelo simplificado propuesto solo se incluye el comportamiento no lineal en las juntas.
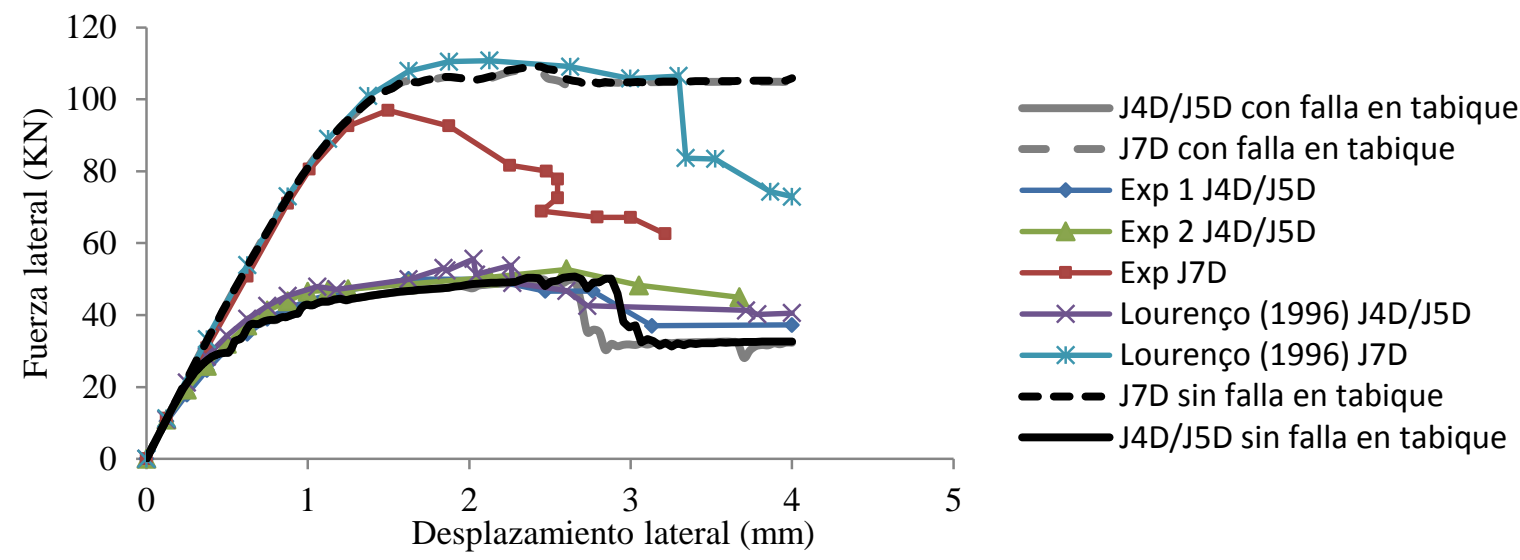

Figura 8. Gráficas carga-desplazamiento para los muros J4D/J5D y J7D (Lourenço, 1996) y lo obtenido con el modelo simplificado propuesto en este estudio.

Se puede apreciar que con el modelo propuesto (ver figuras 9a y 10a) los esfuerzos de compresión obtenidos en la mampostería no alcanzan la resistencia a compresión, de modo que el comportamiento observado, en ningun caso, se vió afectado por el ablandamiento a compresión de la mampostería. En el modelo de Lourenço (ver figura 9b) el esfuerzo de compresión es un poco mayor por lo que se produce aplastamiento en el borde del muro. Por otro lado, la resistencia a corte de la mampostería debió reducirse debido a los niveles de esfuerzo de compresión, ya que de acuerdo al modelo de Lourenço (ecuación 1), para el muro J7D se esperaba una reducción de la resistencia a corte a partir de un esfuerzo de compresión $\sigma_{i}=4.42 \mathrm{~N} / \mathrm{mm}^{2}$, que según los resultados numéricos con el modelo propuesto dichos esfuerzos se presentan para un desplazamiento de $1.1 \mathrm{~mm}$ aproximadamente. Esto coincide con el resultado experimental donde se observa un cambio de pendiente aproximadamente a dicho nivel de deformación. Los modelos numéricos cambian de pendiente poco despues. Con el modelo de Lourenço se aprecia una reducción de la resistencia muy lenta que podría atribuirse a la reducción de la resistencia a corte por compresión, finalmente 
reduce la resistencia a corte en forma abrupta para un desplazamiento de $3.3 \mathrm{~mm}$, que se atribuye al efecto de considerar el agrietamiento a la mitad de las piezas. Con el modelo propuesto el efecto de considerar el agrietamiento a la mitad de las piezas no tuvo el efecto reportado por Lourenço, y no se observa una degradación de la resistencia máxima debido a que se consideró una resistencia a corte constante $\tau_{u}$ a partir de un valor de compresión igual a $\sigma_{i}=\left(\tau_{u}-c\right) / \mu=4.8 \mathrm{~N} / \mathrm{mm}^{2}$.

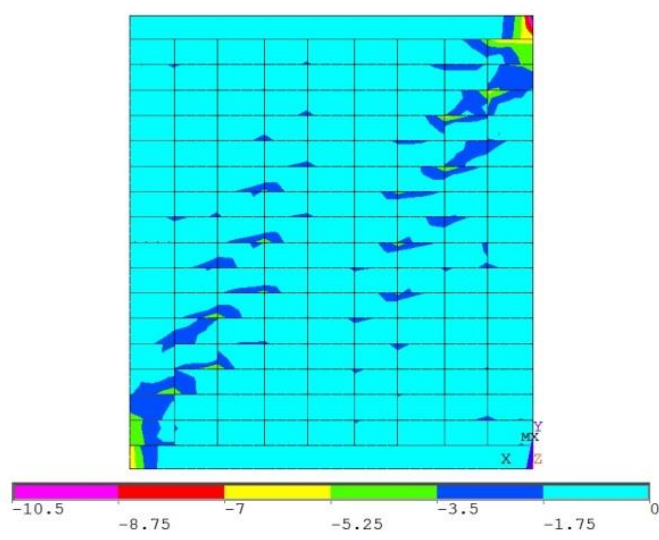

a) Esfuerzo principal de compresión obtenido en este estudio $\sigma_{\min }\left(\mathrm{N} / \mathrm{mm}^{2}\right)$

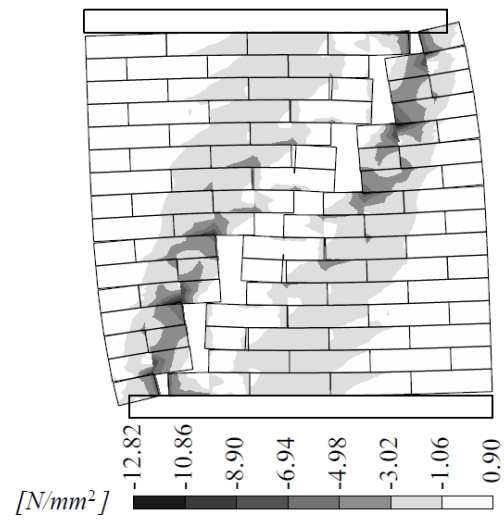

b) Esfuerzo principal de compresión $\sigma_{\min }\left(\mathrm{N} / \mathrm{mm}^{2}\right)$ Lourenço (1996)

Figura 9. Comparación de resultados para los muros J4D/J5D para un desplazamiento lateral de $4 \mathrm{~mm}$.

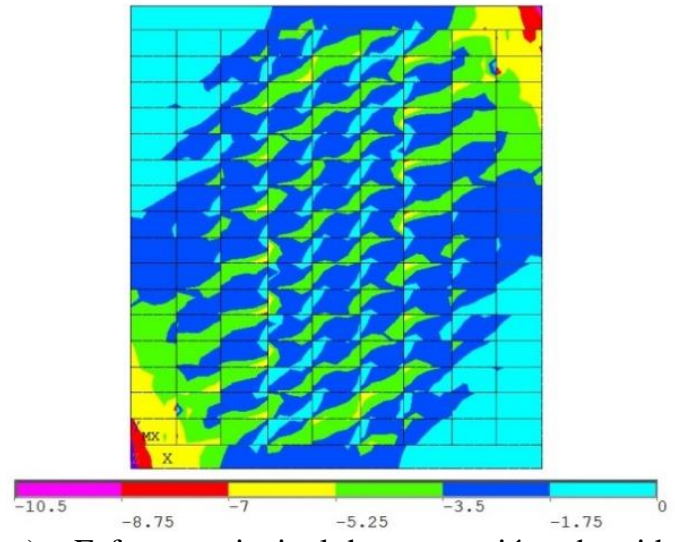

a) Esfuerzo principal de compresión obtenido en este estudio $\sigma_{\min }\left(\mathrm{N} / \mathrm{mm}^{2}\right)$

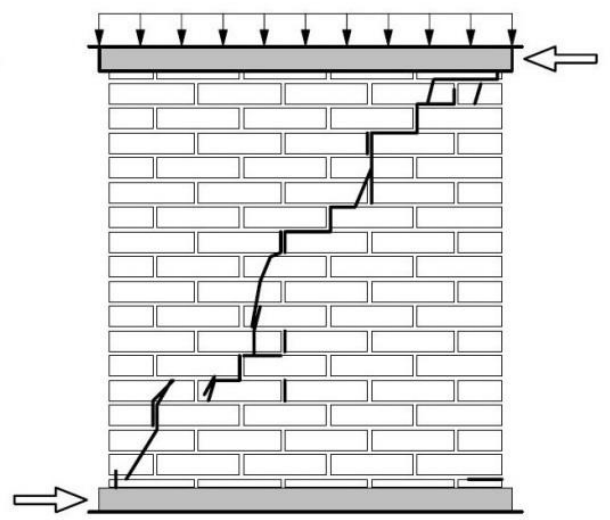

b) Agrietamiento lateral reportado en Lourenço (1996)

Figura 10. Comparación de resultados para el muro J7D para un desplazamiento lateral de $4 \mathrm{~mm}$.

\section{Modelo no lineal de muros de mampostería confinada}

Se modela un muro de mampostería confinada llamado ME5, los resultados experimentales se reportaron en Pérez Gavilán et al. (2013) y Pérez Gavilán et al. (2015). El muro fue sometido a carga axial constante de $0.49 \mathrm{~N} / \mathrm{mm}^{2}$ y carga lateral cíclica reversible. Las dimensiones del muro modelado se muestran en la figura 11. De los ciclos de carga lateral se obtuvieron las envolventes experimentales positiva y negativa que se muestran en la figura 12. Además, se muestra el resultado obtenido numéricamente en este estudio. El muro se construyó con tabiques de barro extruido, por lo tanto se tuvieron que tomar en cuenta los orificios que se presentan en la pieza. Se realizó una homogenización elástica para obtener las propiedades elásticas ortotrópicas equivalentes de un tabique, se utilizó el método descrito en Lizárraga 
(2011). Las dimensiones del tabique utilizado son de 221 de largo por $118 \mathrm{~mm}$ de alto, con un espesor de $113 \mathrm{~mm}$. Los resultados de la homogenización del tabique se muestran en la tabla 3.

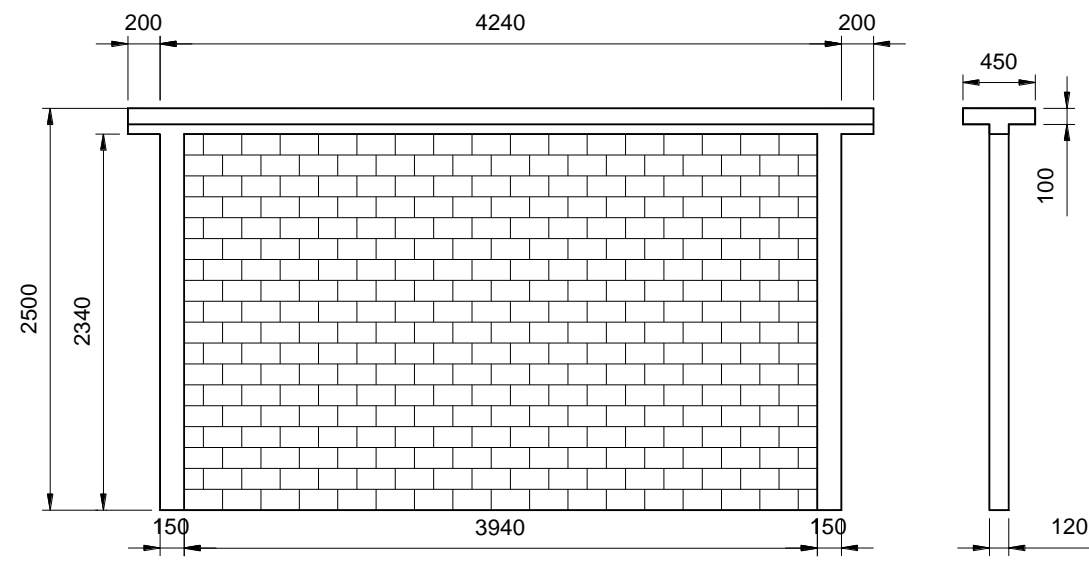

Figura 11. Dimensiones geométricas del muro ME5 (acotación en mm).

Para obtener las propiedades elásticas de la pieza se realizó un modelo 3D del tabique con una malla promedio de $5 \mathrm{~mm}$ utilizando elementos sólidos de 8 nodos (SOLID185) con 3 grados de libertad por nodo (translación en los ejes $\mathrm{x}, \mathrm{y}, \mathrm{z}$ ). De acuerdo a los resultados experimentales se consideró un módulo de elasticidad de la arcilla con que está constituido el tabique de $E_{m a t}=10,612 \mathrm{~N} / \mathrm{mm}^{2}$. Se asignaron distintas propiedades elásticas para modelar las juntas horizontales y verticales, las propiedades de estas interfaces se obtuvieron con las rigideces del material ortotrópico (tabla 3), y con las ecuaciones 3 y 4 utilizando un módulo de elasticidad del mortero de $E_{m}=1,454 \mathrm{~N} / \mathrm{mm}^{2}$ y $v_{m}=0.33$. El módulo de elasticidad del mortero se obtuvo indirectamente con las pruebas de pilas a compresión el procedimiento se describe en Lizárraga (2011). El módulo de cortante del mortero se obtuvo considerando un material isotrópico por lo tanto $G_{m}=547 \mathrm{~N} / \mathrm{mm}^{2}$. Las propiedades elásticas en las interfaces también se muestran en la tabla 3 .

Tabla 3. Propiedades elásticas de la mampostería para el muro ME5

\begin{tabular}{|c|c|c|c|}
\hline \multicolumn{4}{|c|}{$\overline{\text { Pieza }}$} \\
\hline$E_{x}$ & $E_{y}$ & $G_{x y}$ & $v_{x y}$ \\
\hline $2,319 \mathrm{~N} / \mathrm{mm}^{2}$ & $5,805 \mathrm{~N} / \mathrm{mm}^{2}$ & $1,208 \mathrm{~N} / \mathrm{mm}^{2}$ & 0.281 \\
\hline \multicolumn{2}{|c|}{ Junta horizontal } & \multicolumn{2}{|c|}{ Junta vertical } \\
\hline$k_{n}=194 \mathrm{~N} / \mathrm{mm}$ & $k_{s}=100 \mathrm{~N} / \mathrm{mm}^{3}$ & $k_{n}=390 \mathrm{~N} / \mathrm{mm}^{3}$ & $k_{s}=100 \mathrm{~N} / \mathrm{mm}^{3}$ \\
\hline
\end{tabular}

Se asignaron las mismas propiedades inelásticas para las juntas horizontales y verticales. Los parámetros que definen el comportamiento a tensión y a corte en la junta horizontal se obtuvieron de pruebas realizadas previamente en mampostería de block de concreto multiperforado (Lizárraga y Pérez Gavilan, 2013). El valor de la energía de fractura en este caso es de $G_{f}^{I I}=0.4 \mathrm{Nmm}^{-1}$, los coeficientes de fricción inicial y residual asignados en el modelo fueron de $\mu=1.12$ y $\mu_{r 0}=0.89$, y la cohesión $c=0.55 \mathrm{~N} / \mathrm{mm}^{2}$. Para definir $\tau_{u}$ se consideró $f_{m}=11 \mathrm{~N} / \mathrm{mm}^{2}$ y $C_{s}=9$. La resistencia a compresión $f_{m}$ se obtuvo de la resistencia media a compresión de 3 pilas (Pérez Gavilán et al., 2013). Las propiedades inelásticas para el ME5 se resumen en la tabla 4. 
Tabla 4. Propiedades inelásticas para el muro ME5

\begin{tabular}{cccccccc}
\hline Interfaz & \multicolumn{2}{c}{ Tensión } & \multicolumn{6}{c}{ Cortante } \\
\hline & $f_{t}$ & $G_{f}^{I}$ & $c$ & $\mu$ & $\mu_{r}($ ec. 9) & $s($ ec. 11) & $\tau_{u}($ ec. 12) \\
& $\mathrm{N} / \mathrm{mm}^{2}$ & $\mathrm{Nmm}^{-1}$ & $\mathrm{~N} / \mathrm{mm}^{2}$ & - & - & $m m$ & $\mathrm{~N} / \mathrm{mm}^{2}$ \\
\hline Juntas & 0.1 & 0.015 & 0.55 & 1.12 & 0 & 1.46 & 3.67 \\
\hline
\end{tabular}

Las piezas se modelaron con elementos elásticos ortotrópicos, se utilizó una discretización de una longitud igual a la mitad de un tabique. Para representar a la mampostería y al concreto se utilizaron elementos de esfuerzo plano (PLANE183) de 8 nodos con dos grados de libertad en cada nodo (translación en los ejes $\mathrm{x}, \mathrm{y}$ ). Las juntas horizontales y verticales fueron modeladas con elementos de contacto 2D (CONTA172 y TARGE169) de tres nodos con dos grados de libertad en cada nodo (translación en los ejes $\mathrm{x}, \mathrm{y}$ ). Para aplicar la carga lateral se modeló una viga unida en la parte superior del muro (ver figura 13a), se modificó el módulo de elasticidad de ésta viga para dar una rigidez a flexión equivalente a la viga que se utilizó experimentalmente. Se consideró que el concreto permanecía elástico ya que no se afectaba mucho el comportamiento. El módulo de elasticidad del concreto fue de $E_{c}=11,124 \mathrm{~N} / \mathrm{mm}^{2}$ y su relación de Poisson de $v_{c}=0.14$. Debido a que el concreto se modeló elástico, no se incluyó el refuerzo interior en las dalas y castillos.

Se obtuvo con buena aproximación el cambio de rigidez y la carga máxima. La carga promedio máxima experimental registrada fue de $V_{\text {max _exp }}=303 \mathrm{kN}$ mientras que la carga máxima calculada fue de $V_{\max }=296 \mathrm{kN}$, dando un error del $-2 \%$. La rigidez inicial promedio experimental tomada hasta el $40 \%$ de la carga máxima fue de $169 \mathrm{kN} / \mathrm{mm}$, mientras que del análisis numérico se obtuvo una rigidez de $158 \mathrm{kN} / \mathrm{mm}$ dando un error del $-7 \%$.

En la figura 12 se puede observar que el resultado obtenido numéricamente se ajusta con buena aproximación a los resultados reportados experimentalmente. Para una distorsión de aproximadamente $0.08 \%$ se observa experimentalmente un notable cambio de rigidez, mientras que en el modelo numérico la rigidez va cambiando paulatinamente. Para una distorsión cercana a $0.17 \%$ se observa que ocurre la carga lateral máxima en el modelo numérico, a ese nivel de distorsión se presentó la falla escalonada (ver figura 13a), además se presenta inestabilidad numérica en los elementos de contacto. Experimentalmente la distorsión a la cual ocurre la carga máxima fue de $0.2 \%$ para la envolvente negativa y de $0.35 \%$ para la envolvente positiva. En la figura 13 se muestra el patrón de agrietamiento obtenido numéricamente y el obtendio experimentalmente. Se observa que domina el agrietamiento diagonal y la separación del castillo en el modelo numérico se observa como un agrietamiento vertical en el muro ensayado. En la figura 14 se muestran los estados de esfuerzo a corte y los esfuerzos principales a compresión para la carga máxima. Se puede observar cláramente el puntal de compresión (figura 14b), llama la atención que el puntal se desarrolla por arriba de la grieta diagonal. Se puede apreciar el trabajo del castillo para resistir al puntal de compresión mediante la concentración de los esfuerzos cortantes y compresión que finalmente sizallaron el castillo (figura 14a). 


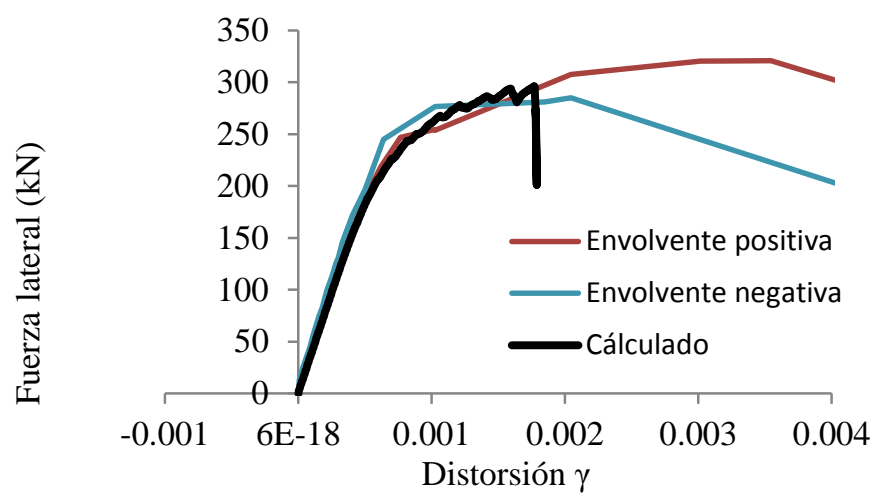

Figura 12. Comparación de las envolventes de carga lateral-distorsión obtenidos numéricamente en este estudio y los reportados experimentalmente (Pérez Gavilán et al., 2013).

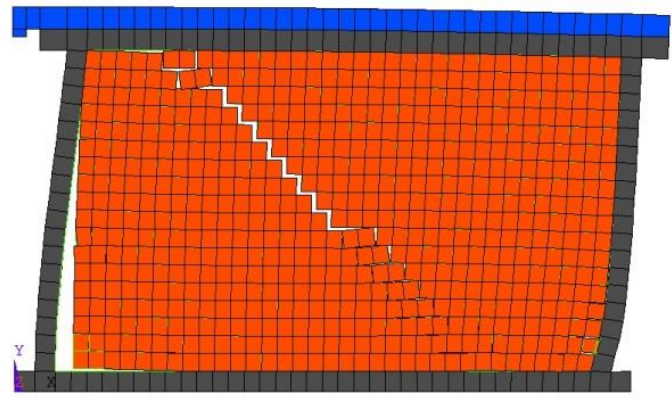

a) Configuración deformada obtenida numéricamente en este estudio para una distorsión de $\gamma=0.0017$

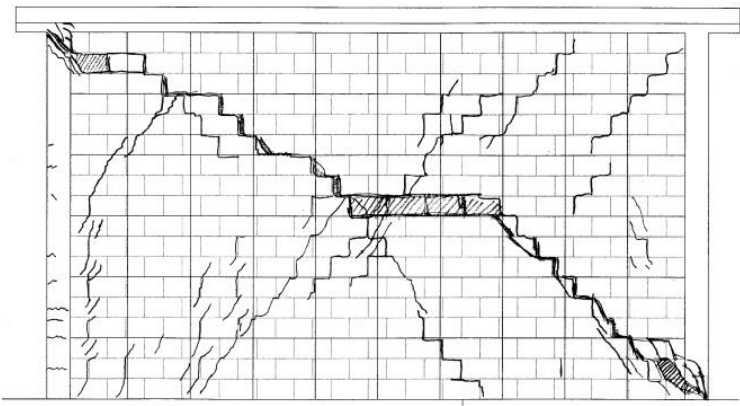

b) Daño presentado experimentalmente (Pérez Gavilán et al., 2013)

Figura 13. Patrón de agrietamiento obtenido.

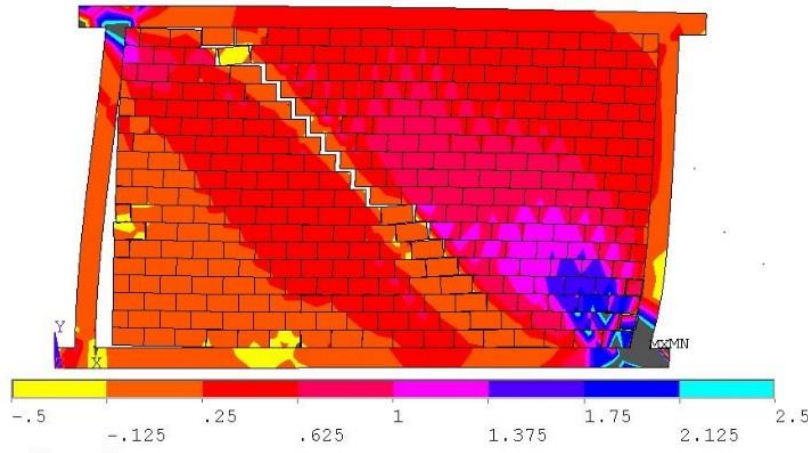

a) Esfuerzo cortante $\left(\mathrm{N} / \mathrm{mm}^{2}\right)$

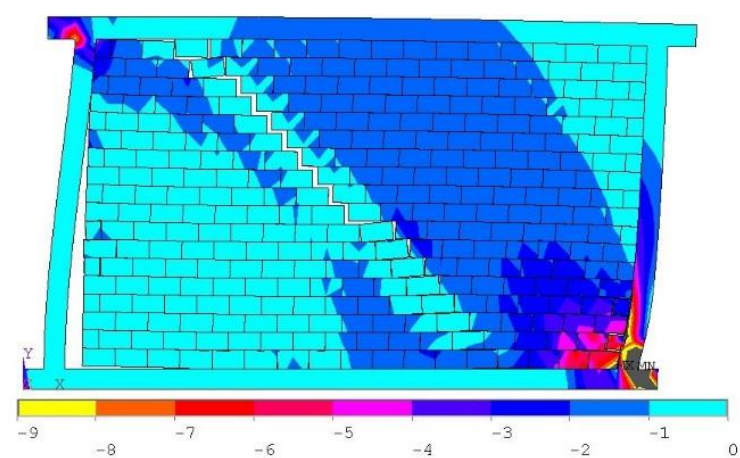

b) Esfuerzo principal de compresión $\left(\mathrm{N} / \mathrm{mm}^{2}\right)$

Figura 14. Esfuerzos obtenidos numéricamente. 


\section{CONCLUSIONES}

De los resultados presentados en este estudio se pueden concluir los siguiente:

- En el modelo utilizado se limita la resistencia a corte a un valor máximo. Se observa que el modelo propuesto no alcanza a degradar la resistencia a corte, lo que se atribuye a este efecto.

- Se puede considerar que tanto los modelos detallados como el micro-modelo simplificado representan de una mejor manera la distribución de esfuerzos a lo largo del muro si se comparan con el modelo homogéneo que da como resultado un valor promedio de los esfuerzos.

- El modelo homogéneo es una buena opción cuando lo que importa es conocer el comportamiento global de un muro; sin embargo, para obtener los parámetros necesarios para la modelación se requiere de ensayes especiales de laboratorio. En futuras investigaciones sería útil comparar el desempeño de estos modelos con los presentados en este artículo.

- La reducción de la resistencia a corte debido a los esfuerzos de compresión no fue significativa en los especímenes con un bajo nivel de carga axial $\left(0.3 \mathrm{~N} / \mathrm{mm}^{2}\right)$. El resultado obtenido limitando la resistencia a corte a un valor máximo (modelo simplificado) fue suficiente para capturar el comportamiento general del muro. Lo anterior también se observó para el caso del muro ME5 con una carga axial de $0.5 \mathrm{~N} / \mathrm{mm}^{2}$.

- Para el muro con una carga axial elevada el efecto de la reducción de la resistencia a corte debida al esfuerzo axial fue mas importante, a juzgar por el resultado experimental. Sin embargo, el modelo que incluye una reducción de forma elíptica de la resistencia con el esfuerzo axial (ecuación 1 Lourenço) redujo la resistencia en forma muy lenta si se compara con lo observado experimentalmente, y la diferencia con el modelo simplificado es poco significativa. Con ambos modelos se pudo estimar una carga resistente similar, y en en ambos casos mayor a la obtenida experimentalmente.

- Para estimar los parámetros de la reducción elíptica es necesario hacer complejos ensayes de pilas con juntas inclinadas o bien no menos complejos ensayes biaxiales. Sin embargo, los resultados muestran que no parece estar justificado dado que la degradación obtenida, con los modelos de otros autores, no logró representar adecuadamente la degradación de la resistencia. Un modelo simplificado como el utilizado aquí da resultados similares.

- De los muros modelados sin confinamiento se puede concluir que la inclusión de la falla a tensión a la mitad de la pieza no cambia significativamente los resultados.

- Con el modelo utilizado se puede representar con buena aproximación el comportamiento del muro de mampostería confinada desde el inicio del agrietamiento hasta la carga máxima; sin embargo, la parte de degradación de resistencia se debe estudiar con más detalle, ya que no fue posible reproducir este comportamiento. Es necesario estudiar más casos con distintos tipos de mampostería y geometrías.

Un análisis detallado de los esfuerzos cortantes en las piezas muestra que estos esfuerzos pueden alcanzar valores muy elevados. En futuras investigaciones deberá revisarse y eventualmente proponerse un modelo para limitar dichos esfuerzos. El limitar dichos esfuerzos puede conducir a una mejor representación de la degradación de la resistencia a corte de los muros.

\section{AGRADECIMIENTOS}

Esta investigación se realizó con el financiamiento de la Dirección General de Asuntos del Personal Académico (DGAPA) de la UNAM proy: IT104112. El autor también agradece a CONACYT por la beca otorgada. 


\section{REFERENCIAS}

Alfano, G, y M A Crisfield (2001), "Finite element interface models for the delamination analysis of laminated composites: Mechanical and computational issues", International Journal for Numerical Methods in Engineering, Vol. 50, pp. 1701-1736.

Ansys (v13), “Ansys Inc”, Theory Reference.

Asteris, P, y A Tzamtzis (2003), “A finite element technique for the analysis of infilled R/C frames”, Ninth North American Masonry Conference, pp. 734-742.

Augenti, N y F Parisi (2011), "Constitutive modelling of tuff masonry in direct shear", Construction and Building Materials, Vol. 25, pp. 1612-1620.

Bazán, E (1980), "Muros de mampostería ante cargas laterales", Tesis de Doctorado, Posgrado en Ingeniería, UNAM.

Casolo, S (2004), "Modelling in-plane micro-structure of masonry walls by rigid elements", International Journal of Solids and Structures, Vol. 41, pp. 3625-3641.

Dhanasekar, M y A W Page (1986), "The influence of brick masonry infill properties on the behaviour of infilled frames", Proceedings of the Institution of Civil Engineers, Vol. 81, No. 4, pp. 593-605.

Gambarotta, L y S Lagomarsino (1997), "Damage models for the seismic response of brick masonry shear walls. Part I: the mortar joint model and its applications", Earthquake Engineering and Structural Dynamics, Vol. 26, pp. 423-439.

Giamundo, V, V Sarhosis, G Lignola, Y Sheng y G Manfredi (2014), "Evaluation of different computational modelling strategies for the analysis of low strength masonry structures", Engineering Structures, Vol. 73, pp. 160-169.

King, G J W y P C Pandey (1978), “The analysis of infilled frames using finite elements”, Proceedings of the Institution of Civil Engineers, Vol. 65, No. 4, pp. 749-760.

König, G (1991), "The state of the art in earthquake engineering research", Experimental and Numerical Methods in Earthquake Engineering, pp. 2-22.

Liaw, T C y C Q Lo (1988), "Multibay infilled frames without shear connectors", ACI Structural Journal, Vol. 85, No. 4, pp. 423-428.

Lizárraga, J F (2011), "Comportamiento de muros de mampostería confinada con distintas configuraciones", Tesis de Maestría, División de Estudios de Posgrado de la Facultad de Ingeniería, UNAM.

Lizárraga, J F y J J Pérez Gavilán (2011), “Análisis no lineal de muros de mampostería confinada utilizando la homogenización del material”, XVIII Congreso Nacional de Ingeniería Sísmica.

Lizárraga, J F y J J Pérez Gavilan (2013), "Propiedades mecánicas de la mampostería para la modelación no lineal”, XIX Congreso Nacional de Ingeniería Sísmica.

Lofti, A W y P B Shing (1994), "Interface model applied to fracture of masonry structures", Journal of Structural Engineering ASCE, Vol. 120, No. 1, pp.63-80.

Lourenço, P B (1996), “Computational strategy for masonry structures”, Tesis de Doctorado, Delft University Press.

Mallick, D V y R T Severn (1967), “The behaviour of infilled frames under static loading”, Proceedings of the Institution of Civil Engineers, Vol. 38, No. 4, pp. 639-656. 
Meli, R y A Reyes (1975), “Comportamiento sísimico de muros de mampostería”, 2da Ed. Corregida y aumentada, Instituto de Ingeniería de la UNAM.

Oliveira, D (2003), "Experimental and numerical analysis of blocky masonry structures under cyclic loading", Tesis de Doctorado.

Orduña, A (1999), "Modelo de muros de mampostería para el análisis no lineal de marcos de concreto reforzado con muros diafragma", Tesis de Maestría, División de Estudios de Posgrado de la Facultad de Ingeniería, UNAM.

Orduña, A y P Lourenço (2005), “Three-dimensional limit analysis of rigid blocks assemblages. Part II: Load-path following solution procedure and validation", International Journal of Solids and Structures, Vol. 42, pp. 5161-5180.

Page, A W (1978), "Finite element model for masonry", Journal of Structural Engineering ASCE, Vol. 104, pp. 1267-1285.

Pérez Gavilán, J J, L Flores y S Alcocer (2013), "Efecto de la esbeltez en la resistencia de muros de mampostería confinada", Revista de Ingeniería Sísmica, Vol. 89, pp. 55-77.

Pérez Gavilán, J J, L Flores y S Alcocer (2015), “An experimental study of confined masonry walls with varying aspect ratio", Earthquake Spectra, Vol. 31, No. 2, pp. 945-968.

Pluijm, R V (1993), "Shear behavior of bed joints", Proceedings of the 6th Canadian Masonry Symposium, pp. 125-136.

Raijmakers, T y A T Vermeltfoort (1992), "Deformation controlled tests in masonry shear walls" (en holandés), Report B-92-1156, TNO-Bouw, Delft, Países Bajos.

Sarhosis, V, K Tsavdaridis y I Giannopoulos (2014), "Discrete element modelling of masonry infilled steel frames with multiple window openings subjected to lateral load variations", The Open Construction and Building Technology Journal, Vol. 8, pp. 93-103.

Stavridis, A y P B Shing (2010), "Finite-element modeling of nonlinear behavior of masonry-infilled RC frames", Journal of Structural Engineering ASCE, Vol. 136, pp. 285-296.

Van Zijl, G (2004), "Modeling masonry shear-compression: Role of dilatancy highlighted", Journal of Engineering Mechanics, Vol. 130, No. 11, pp. 1289-1296.

Vermeltfoort, A y T Raijmakers (1993), "Deformation controlled tests in masonry shear walls, part 2" (en holandés), Report TUE/BKO/93.08, Eindhoven University of Technology, Eindhoven, Países Bajos. 